\title{
Profiling the Urinary Microbiota in Men with Positive versus Negative PD-L1 Expression for Non-muscle Invasive Bladder Cancer
}

\section{Chunxiao Chen}

Nanfang Hospital, Southern Medical University

\section{Zehai Huang}

Nanfang Hospital, Southern Medical University

Pengcheng Huang

Nanfang Hospital, Southern Medical University

Kun Li

The third hospital of mianyang

Jiarong Zeng

Meizhou People's Hospital

Yuehui Wen

Meizhou hospital of TCM

\section{Biao Li}

Nanfang Hospital, Southern Medical University

Jie Zhao

Southern Medical University

Peng Wu ( $\nabla$ doctorwupeng@gmail.com )

Nanfang Hospital, Southern Medical University

\section{Research Article}

Keywords: bladder cancer, urinary tract, microbiota, PD-L1, immune escape

Posted Date: July 28th, 2021

DOl: https://doi.org/10.21203/rs.3.rs-745184/v1

License: (c) (i) This work is licensed under a Creative Commons Attribution 4.0 International License.

Read Full License 


\section{Abstract \\ Background}

Urinary microbiota is associated with the recurrence of bladder cancer, but the underlying mechanism remains unclear. The notion that microbiota can upregulate PD-L1 expression in tumors to promote immune escape have been demonstrated. We hypothesized that the urinary microbiota may be involved in the recurrence and progression of non-muscle invasive bladder cancer (NMIBC) by upregulating the PDL1 expression. For proving this hypothesis, we firstly performed this study to characterize the potential urinary microbial community possibly associated with PD-L1 expression in male patients with NMIBC.

\section{Results}

The subjects (aged 43-79 years) based on their PD-L1 immunohistochemical results were divided into PD-L1-positive group (P group) and PD-L1-negative group (N group) respectively. We observed that $\mathrm{P}$ group exhibited higher species richness and diversity (based on Observed species and Ace index, both $P<$ 0.05). Significantly different composition of urinary microbiota was found between $P$ group and $\mathrm{N}$ group (based on weighted Unifrac and unweighted Unifrac distances metric, both $P<0.05$ ). Enrichment of some bacterial genera (e.g., Leptotrichia, Roseomonas, and Propionibacterium) and decrease of some bacterial genera (e.g.,Prevotella and Massilia) were observed in $\mathrm{P}$ group as compared with $\mathrm{N}$ group. These findings indicate that these genera may affect the expression of PD-L1 through some mechanisms to be studied. PICRUSt analysis showed that several pathways involved in the metabolism of chemical compounds and immune-related disease were enriched in the PD-L1 negative group.

\section{Conclusion}

Our data indicate that urinary microbiota may be an important determinant of PD-L1 expression in male NMIBC patients. The findings of our study may facilitate subsequent study on the role and mechanism of urinary microbiota in the recurrence of NMIBC and may also pave a new way for the better application of PD1 or PD-L1 blockers in bladder cancer in the future.

\section{Background}

Bladder cancer with an estimated 549,000 new cases and 200,000 deaths was the 10 th most common malignancy worldwide according to a 2018 report on the incidence and mortality of 36 cancers in 185 countries [1]. And $75 \%$ of bladder cancers are non-muscle invasive bladder cancer (NMIBC) at initial presentation, among which $\mathrm{Ta}$ and $\mathrm{T} 1$ lesions are generally characterised by a good prognosis and low lethal potential [2], however, their recurrence rates within 5 years are $50-70 \%$ after initial treatment, and $10-30 \%$ of which will progress to muscle-invading tumor [3]. Researchers have long been studying the 
recurrence causes of non-muscle invasive bladder cancer, unfortunately, the exact cause and mechanism of it remain unclear.

Some studies have shown that immune escape might be involved in the recurrence and progression of bladder cancer via the PD-L1 / PD1 pathway [4, 5]. PD-L1 (B7H1) is a member of the B7 family of costimulatory molecules. It mainly promotes apoptosis by combining with programmed death receptors(PD1) expressed on the surface of $T$ cells and $B$ cells, thereby suppressing host immune function and achieving tumor immune escape [6, 7]. Hurwitz ME et a/ reported that PD-L1 expression appears to increase as bladder cancer recurs [5].The recurrence and progression rates of NMIBC are higher in patients with positive PD-L1 expression than that in patients with negative PD-L1 expression. And various factors and mechanisms are involved in the regulation of PD-L1 expression, such as IFNY and microbiota [8-13].Therefore, these factors and mechanisms may promote the recurrence or progression of NMIBC by regulating the expression of PD-L1.

In recent years, the relationship between urinary microbiota and bladder cancer has received widespread attention. Our previous study found that Herbaspirillum, Porphyrobacter and Bacteroides are enriched in bladder cancer patients with high risk of recurrence and progression, which suggests that these genera might be associated with the recurrence of bladder cancer [14]. Zitvogel et a/ reported that oral administration of Lactobacillus after removal of the bladder tumor could reduce the probability of recurrence [15], which further clarified the relationship between microbiota and bladder cancer recurrence. However, the underlying mechanism remains to be further studied. The important roles of microbiota in

multiple systems of human body have been proven, including the immune system [16].And some research have demonstrated that microbiota can upregulate the expression of PD-L1 in tumors and hypersensitivity diseases to promote immune escape or immune tolerance [11-13]. Based on these results, we hypothesized that the urinary microbiota may be involved in the recurrence and progression of NMIBC by up-regulating PD-L1 expression.

In this study, we mainly aimed to characterize urinary microbiota associated with PD-L1 expression in male patients with NMIBC, which may be the basis for subsequent study on the role and mechanism of urinary microbiota in the recurrence of NMIBC and may also provide guidance for the better application of PD1 or PD-L1 blockers in bladder cancer in the future.

\section{Results}

\section{PD-L1 Expression on Tumor Cell Membrane or Tumor-Associated Macrophages (TAMs)}

28 male patients with NMIBC had tumor samples and 16S rRNA gene sequence data to be evaluated for PD-L1 expression on tumor cells or TAMs. Among them, the tumor stage of 13 patients was TaNOM0, and that of 15 patients was T1NOM0. Overall, PD-L1 expression was negative in 19 patients $(67.9 \%)$ and positive in 9 patients (32.1\%, including 6 patients with PD-L1 expression on tumor cells and 3 patients with PD-L1 expression on TAMs). And PD-L1 did not appear in tumor cells and TAMs simultaneously in PD-L1 positive specimens. Out of the 28 male patients with NMIBC, the intensity of PD-L1 was assessed 
and recorded as absent (0) in 19 patients (67.9\%), mild (1+) in 6 patients $(21.4 \%)$, moderate $(2+)$ in 1 patients (3.6\%), and severe (3+) in 2 patients (7.1\%). Representative immunohistochemical staining results are shown in Figure 1.

\section{Demographic Characteristics of Subjects}

A total of 31 mid-stream urine specimens were analyzed, including 11 from male NMIBC patients with positive PD-L1 expression and 20 from male NMIBC patients with negative PD-L1 expression, while 2 specimens in PD-L1-positive group and 1 specimen in PD-L1-negative group were excluded for specimens with too little sequencing reads. Finally, 28 male patients with NMIBC based on their PD-L1 immunohistochemical results were divided into PD-L1-positive group (P group) and PD-L1-negative group (N group) respectively. No statistically significant difference was found in the demographic characteristics between $\mathrm{P}$ and $\mathrm{N}$ group (Table 1). 
TABLE 1. Comparisons of demographic characteristics between PD-L1-positive(P group) and PD-L1negative groups(N group)

\begin{tabular}{|c|c|c|c|}
\hline Demographic characteristics & $P$ group $(n=9)$ & $\mathrm{N}$ group $(\mathrm{n}=19)$ & $P$-value \\
\hline $\operatorname{Age}(y)$ & $67.11(11.48)^{a}$ & $62.05(9.79)^{a}$ & 0.238 \\
\hline Weight(kg) & $63.92(9.03)^{a}$ & $64.79(7.96)^{\mathrm{a}}$ & 0.798 \\
\hline Height(m) & $166(0.06)^{a}$ & $168(005)^{a}$ & 0.270 \\
\hline BMI(kg/m2) & $1.00(0.00)$ & $1.00(0.00)$ & 0.794 \\
\hline Drinking history & $23.16(2.00)^{a}$ & $22.89(2.76)^{\mathrm{a}}$ & 1.000 \\
\hline Hypertension & $11.11 \%(1 / 9)$ & $21.05 \%(4 / 19)$ & 0.677 \\
\hline Diabetes & $44.44 \%(4 / 9)$ & $31.58 \%(6 / 19)$ & 0.290 \\
\hline Hyperlipemia & $33.33 \%(3 / 9)$ & $10.53 \%(2 / 19)$ & 0.095 \\
\hline CHD & $22.22 \%(2 / 9)$ & $0.00 \%(0 / 19)$ & 0.574 \\
\hline $\mathrm{FHC}$ & $22.22 \%(2 / 9)$ & $10.53 \%(2 / 19)$ & 1.000 \\
\hline Smoking index & $0.00 \%(0 / 9)$ & $5.26 \%(1 / 19)$ & 0.841 \\
\hline Percentage of smokers & $200(0,1500)$ & $400(0,800)$ & 0.407 \\
\hline Percentage of multiple tumors & $55.56 \% \varangle 5 / 9 \rrbracket$ & $73.68 \% \otimes 14 / 19 \rrbracket$ & 0.223 \\
\hline Percentage of hypergrading & $22.22 \%(2 / 9)$ & $52.63 \%(10 / 19)$ & 0.114 \\
\hline EORTC_R & $77.78 \% \bigotimes 7 / 9 \rrbracket$ & $42.11 \% \varangle 8 / 19 \rrbracket$ & 0.947 \\
\hline Percentage of HER & $4.44(2.79)^{\mathrm{a}}$ & $4.37(2.83)^{\mathrm{a}}$ & 0.689 \\
\hline EORTC_P & $55.56 \%(5 / 9)$ & $42.11 \%(8 / 19)$ & 0.292 \\
\hline Percentage of HEP & $8.89(4.78)^{a}$ & $6.63(5.36)^{\mathrm{a}}$ & 0.435 \\
\hline Percentage of high invasive risk & $66.67 \%(6 / 9)$ & $47.37 \%(9 / 19)$ & 0.230 \\
\hline \multirow[t]{2}{*}{ Percentage of T1N0M0 } & $88.89 \%(8 / 9)$ & $63.16 \%(12 / 19)$ & 0.114 \\
\hline & $77.78 \% \bigotimes 7 / 9 \rrbracket$ & $42.11 \% \varangle 8 / 19 \rrbracket$ & \\
\hline \multicolumn{4}{|c|}{$\begin{array}{l}\text { Data are presented as mean (SD) or median (first quartile to the third quartile) for continuous } \\
\text { variables or } n(\%) \text { for counting data. a indicates that the datum is subject to normal distribution and } \\
\text { homogeneity of variance test and is presented as mean (SD). Multiple tumors refer to the number of } \\
\text { bladder tumor } \geq 2 \text {. Abbreviations: BMI, body mass index; CHD, coronary atherosclerotic heart disease; } \\
\text { FHC, family history of cancer; EORTC, European Organization Research and Treatment of Cancer } \\
\text { Scoring system, _R refer to recurrence and _P refer to progression; HER, recurrence score of } \\
\text { EORTC } \geq 5 ; \mathrm{HEP} \text {, progression score of EORTC } \geq 7 \text {. }\end{array}$} \\
\hline
\end{tabular}

\section{Sequencing Data, Alpha, Beta Diversity and Alpha Rarefaction Curves}


Reads that contain $50 \%$ of base whose quality value is less than 20 or that contain $65 \%$ of base whose quality value is less than 30 were removed. A total of 962452 reads were obtained from 28 samples after the removal of primer and chimera. The median number of reads in PD-L1 positive group was 26,335 , and in the PD-L1 negative group was $34952(P=0.676)$. And the reads were classified into 2,124 OTUs which were used for downstream analysis. More OTUs were identified in urine from $P$ group, with an average of 215 OTUs in $\mathrm{P}$ group and 141 OTUs in $\mathrm{N}$ group $(P=0.029)$. The number of reads and OTUs for each sample are shown in Supplementary Table $S 1$. The Ace index and Observed species were significantly higher in $\mathrm{P}$ group than in $\mathrm{N}$ group $(P<0.05$; Figures 2A, B). While no differences were found in the Chao1 index (Figure 2C), Shannon index (Figure 2D) and Simpson index (Figure 2E) between P group and N group. Rarefaction curves were constructed for different groups showing P group was much more diverse than $\mathrm{N}$ group (Figure 2F).

In order to further clarify the relationship between PD-L1 expression and urinary microbiota, we divided $\mathrm{P}$ group into $\mathrm{C}$ group (with mild PD-L1 expression) and D group (with moderate or severe PD- L1 expression) based on the expression intensity of PD-L1. Interestingly, we found that as the expression intensity of PD-L1 increased, the bacterial richness showed an increasing trend (Supplementary Figure S1), though no statistical difference was found between the two groups. Among PD-L1 positive group, urinary microbiota of patients with $\geq 5 \%$ of tumor cells membrane staining had higher diversity than those of patients with $₫ 5 \%$ of tumor cells membrane staining (Supplementary Figure S2).

To measure the similarity of urine microbial communities between $\mathrm{P}$ group and $\mathrm{N}$ group, we performed the unconstrained principal coordinate analysis (PCoA) based on Bray Curtis, weighted UniFrac, and unweighted UniFrac distance metrics. We found that the composition of urinary microbiota in $\mathrm{P}$ group differed from that in $\mathrm{N}$ group. The PCoA plots based on weighted Unifrac and unweighted Unifrac distances revealed that the urinary microbiota of $\mathrm{P}$ group and $\mathrm{N}$ group formed two distinct clusters (Figures 3A, B). Besides, we detected 643 OTUs were enriched in P group, 934 OTUs were enriched in N group and with 547 OTUs were shared between two groups (Figure 3C).

\section{Relative Abundance of Urinary Bacteria in $\mathrm{P}$ group and $\mathrm{N}$ group}

At phylum level, the urinary microbiota was dominated by Firmicutes (22.8\% P group, $34.4 \% \mathrm{~N}$ group) and Proteobacteria (21.1\% P group, 25.8\% N group), followed by Actinobacteria (6.6\% P group, $4.0 \% \mathrm{~N}$ group) and Bacteroidetes (1.1\% P group, 9.7\% N group) (Table 2, Figure 4A). The urinary microbial composition of different groups at class, order, family and genus level were exhibited in Figures 4B-E. The genera compositions of all samples were exhibited in Figure 4F. Though the relative abundance of so many microbes at various taxonomic level differed between $\mathrm{P}$ group and $\mathrm{N}$ group, only five taxa including Bacteroidetes $(P=0.017)$, Bacteroidia $(P=0.022)$, Bacteroidales $(P=0.025)$, Prevotellaceae $(P=0.028)$ and Prevotella $(P=0.04)$ were present at higher relative abundance in $\mathrm{N}$ group and one genus called Leptotrichia had higher abundance in $\mathrm{P}$ group $(P=0.001)$, when the Metastats algorithm was used for comparison of the relative abundance of bacteria and the relative abundance threshold of microbes was set at $0.1 \%$ (Table 2 ). 
Page $7 / 25$ 
TABLE 2. Comparison of relative abundance of urinary microbiota between PD-L1 positive(P group) and PD-L1 negative groups(N group).

\begin{tabular}{|c|c|c|c|c|}
\hline Таха & $\mathrm{N}$ group & & P group & $P$-vlaue \\
\hline \multirow[t]{4}{*}{ Phylum } & Firmicutes & 34.438 & 22.828 & Ns \\
\hline & Proteobacteria & 25.756 & 21.077 & Ns \\
\hline & Bacteroidetes & 9.662 & 1.130 & 0.017 \\
\hline & Actinobacteria & 4.012 & 6.631 & Ns \\
\hline \multirow[t]{8}{*}{ Class } & Bacteroidia & 9.334 & 0.856 & 0.022 \\
\hline & Betaproteobacteria & 4.557 & 2.234 & Ns \\
\hline & Negativicutes & 3.230 & 2.353 & Ns \\
\hline & Actinobacteria & 3.085 & 6.488 & Ns \\
\hline & Bacilli & 27.572 & 19.562 & Ns \\
\hline & Unclassified & 22.313 & 44.950 & Ns \\
\hline & Gammaproteobacteria & 19.051 & 16.725 & Ns \\
\hline & Alphaproteobacteria & 1.765 & 2.012 & Ns \\
\hline \multirow[t]{10}{*}{ Order } & Enterobacteriales & 9.744 & 6.043 & Ns \\
\hline & Bacteroidales & 9.316 & 0.853 & 0.025 \\
\hline & Pseudomonadales & 5.051 & 6.358 & Ns \\
\hline & Pasteurellales & 3.973 & 1.995 & Ns \\
\hline & Burkholderiales & 3.477 & 2.042 & Ns \\
\hline & Selenomonadales & 3.226 & 2.352 & Ns \\
\hline & Unclassified & 23.076 & 46.121 & Ns \\
\hline & Lactobacillales & 16.826 & 11.731 & Ns \\
\hline & Bacillales & 10.619 & 7.522 & Ns \\
\hline & Corynebacteriales & 1.928 & 4.426 & Ns \\
\hline \multirow[t]{5}{*}{ Family } & Enterobacteriaceae & 8.580 & 6.001 & Ns \\
\hline & Prevotellaceae & 7.980 & 0.761 & 0.028 \\
\hline & Staphylococcaceae & 7.876 & 7.288 & Ns \\
\hline & Unclassified & 31.822 & 46.981 & Ns \\
\hline & Pasteurellaceae & 3.966 & 1.995 & Ns \\
\hline
\end{tabular}




$\begin{array}{llll}\text { Veillonellaceae } & 2.603 & 2.347 & \text { Ns } \\ \text { Moraxellaceae } & 2.510 & 5.147 & \mathrm{Ns} \\ \text { Comamonadaceae } & 2.452 & 1.804 & \mathrm{Ns} \\ \text { Pseudomonadaceae } & 2.335 & 1.117 & \mathrm{Ns} \\ \text { Streptococcaceae } & 10.553 & 10.664 & \mathrm{Ns} \\ \text { Corynebacteriaceae } & 1.788 & 4.296 & \mathrm{Ns} \\ \text { Escherichia-Shigella } & 8.167 & 4.840 & \mathrm{Ns} \\ \text { Prevotella } & 8.060 & 0.672 & 0.040 \\ \text { Staphylococcus } & 7.187 & 6.941 & \mathrm{Ns} \\ \text { Unclassified } & 36.701 & 49.489 & \mathrm{Ns} \\ \text { Haemophilus } & 3.899 & 1.983 & \mathrm{Ns} \\ \text { Pseudomonas } & 2.306 & 1.106 & \mathrm{Ns} \\ \text { Streptococcus } & 10.433 & 10.485 & \mathrm{Ns} \\ \text { Delftia } & 1.817 & 1.475 & \mathrm{Ns} \\ \text { Veillonella } & 1.727 & 2.213 & \mathrm{Ns} \\ \text { Enhydrobacter } & 1.332 & 3.560 & \mathrm{Ns} \\ \text { Leptotrichia } & 0.000 & 0.205 & 0.001\end{array}$

Data were displayed as mean percentage; For taxa with $P$-value $<0.05$, the taxa shown in the table are those with relative abundance greater than $0.1 \%$. For taxa without significant difference, only taxa with relative abundance greater than $1 \%$ are shown in the table; Ns, not significant (based on $P<$ 0.05).

For the OTUs, we defined the core microbes as the OTUs with relative abundance higher than 0.002 resulting in 52 OTUs altogether. The core microbes of each group were displayed by taxonomic dendrogram (Figure 5). The percentages of the total community covered by the core microbes ranged from $85.9 \%$ (N group) and $87.1 \%$ (P group). And the distribution of core microbes revealed marked changes between the bacterial composition of the different groups. Enrichment of some core microbes (e.g., Ureaplasma, Enhydrobacter, Mucispirillum) and decrease of some core microbes (e.g., Prevotella, Haemophilus, Lactobacillus) were observed in male NMIBC patients with positive PD-L1 expression when compared to PD-L1 negative group.

\section{Specific Taxa Associated With PD-L1 Expression}

The specific microbial taxa associated with PD-L1 expression were identified by using LEfSe algorithm. The results showed that Corynebacteriale, Corynebacteriaceae, Corynebacterium_1, uncultured_Corynebacterium_sp, Propionibacterium, Dermabacteraceae, Brachybacterium, Roseomonas, 
Rhodospirillaceae, Pigmentiphaga were present at significantly higher compositional abundances in $\mathrm{P}$ group compared with $\mathrm{N}$ group, whereas the relative abundance of Massilia, Oxalobacteraceae, Firmicutes was higher in $\mathrm{N}$ group (Figures 6A, B). Heat tree was used to illustrate the taxonomic differences between $\mathrm{N}$ group and $\mathrm{P}$ group (Figure $6 \mathrm{C}$ ). Similarly, in $\mathrm{P}$ group, Roseomonas was enriched in the group with higher PD-L1 expression (group F, PD-L1 $12 \%$ ) and Prevotella had higher abundance in the group with lower PD-L1 expression (group 0, 1\% SPD-L1<5\%) (Supplementary Figure S3).

\section{Potential Functional Pathways Associated With PD-L1 Expression}

The functional pathways of different groups were predicted by PICRUSt based on the microbial community profiles. Overall, the functional profiles present in patients with positive PD-L1 expression and negative PD-L1 expression could not be distinguished clearly (Figure 7A). The ATP-binding cassette transporters associated pathway was significantly enriched in NMIBC patients with positive PD-L1 expression, while the predicted KEGG pathways significantly enriched in NMIBC patients with negative PD-L1 expression included Systemic lupus erythematosus, Transcription machinery, Metabolism of Terpenoids and Polyketides, Selenocompound metabolism , Base excision repair and Nucleotide excision repair (Figure 7B).

\section{Discussion}

In this study, we used 16S rRNA gene sequencing and PD-L1 immunohistochemistry to preliminarily explore the relationship between urinary microbiota and PD-L1 expression in male patients with NMIBC. Higher urinary microbial diversity was found in male patients with positive PD-L1 expression. And through subgroup analysis, we found that with the increase of PD-L1 expression intensity, the diversity of urinary microbiota also showed an increasing trend. In addition, 17 taxa were increased and 3 taxa were reduced in patients with PD-L1 positivity compared with patients with negative PD-L1 expression. The result also demonstrated that higher PD-L1 expression was related to the enrichment of Roseomonas, which suggests that this is a genus worthy of further study.

Over the past decade, the microbiota in different parts of human body have been characterized and some microbiota-related mechanisms which can directly impact human health have begun to be identified [17]. Developing diagnostics and therapeutics that utilize those connections between the microbiome and human health is the next frontier for translational microbiome science [18]. However, the falsifiable hypothesis needs to be discovered through observational studies before performing a randomized validation experiment. In this exploratory study, we found the associations between urinary microbiota and PD-L1 expression in patients with NMIBC and some genera related to PD-L1 expression which may be isolated by bacterial culture technique and experimentally verified for their role in regulating the expression of PD-L1. The findings of this study may be the basis for the subsequent study on the role and mechanism of urinary microbiota in the recurrence of NMIBC and may also provide guidance for the better application of PD1 or PD-L1 blockers in bladder cancer in the future. 
The role of PD-L1 / PD1 mediated immune escape in bladder cancer recurrence and progression has long been studied [4,5], which has facilitated the development of clinical trials of PD-L1 / PD1 inhibitors in NMIBC and the FDA approval of 5 PD-L1/PD1 inhibitors for the treatment of metastatic bladder cancer [19]. And in order to improve the response rate and reduce drug-related adverse reactions, PD-L1 / PD1 inhibitors are offered to patients with bladder cancer depending on the PD-L1 status [20], which indicates that the PD-L1 status is still one of the main factors that determine the efficacy of PD1 / PD-L1 inhibitors. Therefore, exploring factors affecting the expression of PD-L1 may help improve the therapeutic effect of PD-L1/PD1 inhibitors. Groeger S et a/ have reported that Porphyromonas gingivalis and its total membrane fraction can upregulate PD-L1 expression in vitro [11, 12]. In addition, microbiota can also promote immune tolerance to allergens in neonates via PD-L1 [13]. And the concept that microbiota can modulate the clinical response to PD1/PD-L1 inhibitors has been confirmed in experimental studies [21, 22], though the underlying mechanisms are still unknown. Since previous studies and ours have shown that there is a correlation between the microbiota and PD-L1 expression, suggesting that the microbiota may affect the therapeutic effect of PD1/PD-L1 inhibitors by regulating PD-L1 expression. If the role of urinary microbiota in PD-L1 expression is confirmed in the future, urinary microbiota might be a target for enhancing clinical responses to PD1/PD-L1 inhibitors in the treatment of bladder cancer or other urinary tract tumors.

Increased microbial diversity was observed both in bladder cancer patients and NIMBC patients with high risk of recurrence and progression [14], which was consistent with the results from Hai Bi group [23]. In present study, significantly higher bacterial diversity in the PD-L1 positive group was also found, which suggests that higher bacterial diversity may be combined with PD-L1 or used as a substitute for the prediction of high risk of recurrence and progression in NMIBC. Despite the inter-individual differences in the microbiota composition, the urinary microbiota of individuals in the same group still have similarities, as shown in the PCoA analysis that clustered PD-L1 positive group and PD-L1 negative group separately (Figs. $3 \mathrm{~A}$ and $3 \mathrm{~B}$ ), indicating the possibility of a common urinary microbiota disorder associated with PDL1 expression.

In our study, we found that the Leptotrichia was enriched in PD-L1 positive male patients with NMIBC and Prevotella was enriched in PD-L1 negative group. Fusobacterium and Leptotrichia are relatively closelyrelated organisms. They have been isolated from periodontal lesions and a diversity of genitourinary and gastrointestinal abscesses, and have been suggested to be potential emerging pathogens [24]. Studies have demonstrated that Leptotrichia may be involved in the occurrence and development of several carcinoma, such as gastric cancer, colon cancer, pancreatic cancer and etc [25-29]. Jang et al reported IL-6 and IL-8 were strongly induced by Leptotrichia wadei [30], and IL 6 is an inflammatory cytokine involved in various biological processes, including dysimmune diseases and cancers [31, 32]. Chan et al have demonstrated that Pro-inflammatory cytokines such as IFNy and IL-6, can promote the expression of PD-L1 to achieve tumor immune escape [8,33], which is consistent with our results. However, whether Leptotrichia can induce the expression of PD-L1 in bladder cancer and its related mechanisms need further confirmation in vivo and in vitro. As for Prevotella, Calcinotto A and collaborators recently reported that the abundance of Prevotella histicola and Prevotella melaninogenica in the guts of preclinical 
models and humans or their colonization of the mouse gut is associated with decreased proinflammatory Th17 cells in the intestine and bone marrow, leading to delayed multiple myeloma progression [34].On the contrary, Prevotella heparinolytica accelerated the progression of multiple myeloma in mice by inducing the local differentiation of Th17 cells that migrated to the bone marrow and supported plasma cell survival and Prevotella spp. also can sustain the protumorigenic effect of dextran sodium sulfate by favoring Th17 and Th1 immunity in the colon [34,35].All together, data from malignancy patients and murine experimental models converge to support the perspective that Prevotella may participate in the progression of malignant tumors through Th17 cells, but different strains of Prevotella may play an opposite role.

Roseomonas and Propionibacterium were found in higher abundances in PD-L1 positive group than in PD-L1 negative group. Roseomonas mucosa may directly influence atopic dermatitis and provide clinical benefit through multiple mechanisms, including innate/adaptive immune balance [36]. While Propionibacterium strain, P. UF1, can safeguard against proinflammatory diseases by increasing the frequency of colonic Th17 and Treg cells [37]. These studies, together with ours, indicate that these two genera may affect the course of human disease by regulating host's immunity.

ATP-binding cassette transporters, involved in a plethora of cellular tasks, including antibiotic and multidrug resistance, immunity, etc [38], was enriched in PD-L1 positive group. While PD-L1 negative group has higher KEGG pathway related to systemic lupus erythematosus. In systemic lupus erythematosus, nucleic acid-containing immune complexes can trigger production of type I IFN which in turn induce PD-L1 and NOS2 expression in both tumor and dendritic cells [39, 40]. Based on these results, urinary microbiota may regulate the immune function in male patients with NMIBC through these KEGG metabolic pathways. And subsequent studies should be focused on relevant metabolic pathways and important metabolites.

This study does have limitations. First of all, the causal relationship between the urinary microbiota and PD-L1 expression can not be determined by this observational study, which requires further prospective studies and experiments in vivo and in vitro to verify. Secondly, although it is a far less invasive method than suprapubic aspiration or catheterization and therefore suitable for use in daily practice, mid-stream urine specimens collected by the clean catch method may be contaminated by microbiota in the urethra or surrounding the urethral orifice. Last but not least, 16S rRNA gene sequencing may reliably characterize microbiota to the genus level but cannot identify bacteria well at species level and detect viruses or fungi. However, its low cost and easy sampling make 16S rRNA gene sequencing always a pilot in metagenome and other microbiota-related research.

\section{Conclusions}

This is the first study to profile urinary microbiota associated with the expression of PD-L1 in male patients with NMIBC. Our study shows that urinary microbiota is associated with PD-L1 expression in male patients with NMIBC, but the exact relationship remains to be further studied. A defined association 
between urinary microbiota and PD-L1 expression may facilitate subsequent study on the role and mechanism of urinary microbiota in the recurrence of NMIBC and may also provide guidance for the better application of PD1 or PD-L1 blockers in bladder cancer in the future.

\section{Methods}

\section{Study design, subjects recruitment and samples collection}

The aim of this cross-sectional study was to characterize urinary microbiota associated with PD-L1 expression in male patients with NMIBC. Urine specimens and cancerous tissues were collected from male patients with NMIBC who were admitted to Nanfang Hospital in China between March 2017 and June 2019. Eventually, twenty-eight subjects histologically confirmed as non-muscle invasive bladder cancer, aged from 43 to 79 years, were enrolled for the study. The urine specimens were collected prior to the prescription of antibiotics and operation for bladder cancer. Exclusion criteria for the participants included prior known sexually transmitted infection, current urinary tract infection (based on urine dipstick or standard cultivation) or antibiotic consumption within 1 month for any reason. All participants were required to finish a questionnaire to collect their demographic and medical information. Following the Medicine Institutional Review Board of Southern Medical University approval (ethical code: NFEC2020-045) and the Declaration of Helsinki principles, all participants gave written consent for their information, urine and tissue collection with analysis for research purposes.

All urine specimens for urinalysis were obtained by the mid-stream clean catch method with the guidance of urotherapy nurses, then immediately shipped on cold pack to laboratory within an hour for centrifugation at $16,000 \mathrm{~g}$ for $10 \mathrm{~min}$ at $4^{\circ} \mathrm{C}$. The precipitates were stored at -80 degrees Celsius until further processing.

\section{DNA Isolation and 16S rRNA Gene Sequencing}

To avoid contamination, DNA was extracted using the DNeasy Blood and Tissue Kit following the cultured cells protocol provided by the manufacturer (Qiagen, Germany) in a laminar flow hood. The Nanodrop ND-1000 spectrophotometer (Thermo Electron Corporation, USA) was used for the measurement of extracted DNA concentration. 16S rDNA sequences of the genomic DNA isolated from the urine specimens was amplified with PCR using primer sets specific for V3-V4 regions (314F: 5'NCCTACGGGNGGCWGCAG-3'; and 805R: 5'NGACTACHVGGGTATCTAATCC-3'). We added extraction negative controls (no urine) and PCR negative controls (no template) to evaluate the presence of contaminating sequences in reagents. Unincorporated nucleotides and primers were eliminated using the Qiaquick PCR purification kit (Qiagen, Valencia, USA) to obtain the final PCR purified products. After normalization to equal DNA concentration, the purified samples were sequenced using the Illumina Miseq sequencer (Illumina, USA). The 16S rRNA gene sequence data reported in this paper have been deposited in the Genome Sequence Archive (Genomics, Proteomics \& Bioinformatics 2017) in BIG Data Center (Nucleic Acids Res 2018), Beijing Institute of Genomics (BIG), Chinese Academy of Sciences, under accession numbers CRA002985, CRA002985 that are publicly accessible at https://bigd.big.ac.cn/gsa. 


\section{Immunohistochemistry}

Immunohistochemical detection of PD-L1 was performed on formalin-fixed paraffin-embedded tissue using a mouse monoclonal anti-PD-L1 antibody (405.9A11, dilution1:200, Cell Signaling Technology) and following the protocol provided by the manufacturer. For each sample, the membranous expression of PD-L1 in tumor cells or Tumor-Associated Macrophages (TAMs) was determined and scored in blinded manner with respect to clinical data by two independent genitourinary pathologist. PD-L1 positivity was defined by the presence of tumor cells or TAMs with membrane staining. PD-L1 expression in tumor cells or TAMs was scored as the percentage of stained cells. And the intensity of PD-L1 was assessed and recorded as absent (0), mild (1+), moderate (2+) and severe (3+).

\section{Statistical Analysis}

\section{Clinical Data Analysis}

Normal distributions of the data were estimated with Shapiro-Wilk test and homoscedasticity of variances was checked using Levene test. The differences between groups were tested using Student's $t$ test for measurement data subject to normal distribution and homogeneity of variance and Fisher's Exact Test for counting data. While Mann-Whitney $U$ test was used for the statistical analysis of ranked data or measurement data that did not conform to normal distribution or homogeneity test of variance.

Statistical analysis was performed using SPSS statistics version 24 (IBM corp, Amonk, NY, USA). All tests were two sided and $P<0.05$ was considered statistically significant.

\section{Bioinformatics analysis}

In order to obtain clean reads, the raw data of $16 \mathrm{~S}$ rRNA gene sequences were filtered using QIIME [41] to eliminate reads with adapter pollution and low quality. Metadata are given in Supplementary Table S2. The paired-end reads were merged with their overlap using standardized bioinformatic pipeline to attain complete sequence which were clustered into operational taxonomic units (OTUs) with $97 \%$ similarity and the representative sequences were picked by UPARSE [42-44]. Subsequently, UCHIME method in VSEARCH (v2.3.4) was used for the detection and removal of chimera $[45,46]$.The representative sequence from each clustered OTU was aligned to the SILVA database and the Greengenes database and classified with the RDP classifier to achieve species annotation [47-49].

Observed Species, Chao1, Ace, Shannon and Simpson indices were calculated using QIIME to evaluate alpha diversity. Among them, the Observed Species, Chao1 and Ace indices are mainly used to assess the species richness of samples, while Shannon and Simpson indices mirror both species richness and evenness. The difference of alpha diversity between groups was evaluated by Mann-Whitney U Test (group number= 2) or Kruskal-Wallis test (group number > 2) using SPSS statistics version 24 (IBM corp, Amonk, NY, USA) or R software. The Bray Curtis, weighted UniFrac and unweighted UniFrac distances were calculated for the comparison of Beta-diversity of different groups. Three-dimensional plots were generated based on Principal coordinate analysis (PCOA) of these distance matrices in QIIME for the 
visual clustering of the bacterial communities. To statistically support the differences in beta-diversity between groups, these distances of different groups were compared using Adonis test or ANOSIM test.

Average distribution of major taxa was represented by circlize graphs which were drawn with the circlize package in R. And taxonomic dendrogram was generated with one representative sequence of each OTU using Unipro UGENE and displayed with the use of iTOL(Interactive Tree Of Life) [50].To detect significantly different bacteria between groups, the relative abundance of bacteria were compared using Metastats. Taxa summaries were input into Linear discriminant analysis effect size (LEfSe) via the Huttenhower Lab Galaxy Server for further screening of significantly different bacteria between groups at different taxonomic level [51]. In the settings of LEfSe, the significantly specific bacteria were identified using the Mann-Whitney $U$ test, and their effect size were estimated via linear discriminant analysis (LDA) of which the threshold for discriminative features was 2.0. While heat tree was generated with the workflow provided by Chong et al [52] and the use of MicrobiomeAnalyst (https://www.microbiomeanalyst.ca).The functional pathways were predicted based on microbiota composition data via Phylogenetic Investigation of Communities by Reconstruction of Unobserved States (PICRUSt) [53]. And the Predicted KEGG pathways of different groups were compared using STAMP (version 2.1.3) [54].

\section{Abbreviations}

NMIBC: non-muscle invasive bladder cancer; PD-L1: Programmed Cell Death 1 Ligand 1; PD1: Programmed Cell Death Protein 1; TAMs: Tumor-Associated Macrophages; PCoA : principal coordinate analysis; LEfSe: linear discriminant analysis (LDA) effect size; PICRUSt: Phylogenetic Investigation of Communities by Reconstruction of Unobserved States; PCR: Polymerase chain reaction; OTUs: operational taxonomic units; QIIME: Quantitative Insights Into Microbial Ecology

\section{Declarations}

\section{Acknowledgments}

The authors are grateful to all the participants.

\section{Authors' contributions}

PW, CC, JZ: conception and design; $\mathrm{CC}, \mathrm{ZH}, \mathrm{PH}, \mathrm{KL}, \mathrm{JRZ}, \mathrm{YW}, \mathrm{BL}$ : acquisition of data; $\mathrm{CC}, \mathrm{PH}, \mathrm{KL}$ : analysis and interpretation of data; CC, ZH: drafting of the manuscript; PW, JZ, JRZ: critical revision of the manuscript for important intellectual content; $\mathrm{CC}, \mathrm{PH}, \mathrm{KL}$ : statistical analysis; $\mathrm{PW}$, JZ: obtaining funding; PW, JZ: supervision. All authors revised the draft and approved the final version of the manuscript.

\section{Funding}


This study was supported by the Natural Science Foundation of Guangdong Province (grant no. 2021A1515012262), the Natural Science Foundation of Guangdong Province (grant no.

2020A1515011339) and the National Natural Science Foundation of China (grant no. 81870522).

\section{Availability of data and materials}

The 16S rRNA gene sequence data reported in this paper have been deposited in the Genome Sequence Archive (Genomics, Proteomics \& Bioinformatics 2017) in BIG Data Center (Nucleic Acids Res 2018), Beijing Institute of Genomics (BIG), Chinese Academy of Sciences, under accession numbers CRA002985, CRA002985 that are publicly accessible at https://bigd.big.ac.cn/gsa.

\section{Ethics approval and consent to participate}

Following the Medicine Institutional Review Board of Southern Medical University approval (ethical code: NFEC-2020-045) and the Declaration of Helsinki principles, all participants gave written consent for their information, urine and tissue collection with analysis for research purposes.

\section{Consent for publication}

Not applicable.

\section{Competing interests}

The authors declare that they have no competing interests.

\section{References}

1. Bray F, Ferlay J, Soerjomataram I, Siegel RL, Torre LA, Jemal A: Global cancer statistics 2018 : GLOBOCAN estimates of incidence and mortality worldwide for 36 cancers in 185 countries. CA: a cancer journal for clinicians 2018, 68(6):394-424.

2. de Braud F, Maffezzini M, Vitale V, Bruzzi P, Gatta G, Hendry WF, Sternberg CN: Bladder cancer. Critical reviews in oncology/hematology 2002, 41(1):89-106.

3. Kamat AM, Hahn NM, Efstathiou JA, Lerner SP, Malmström PU, Choi W, Guo CC, Lotan Y, Kassouf W: Bladder cancer. Lancet (London, England) 2016, 388(10061):2796-2810.

4. Fukumoto K, Kikuchi E, Mikami S, Hayakawa N, Matsumoto K, Niwa N, Oya M: Clinical Role of Programmed Cell Death-1 Expression in Patients with Non-muscle-invasive Bladder Cancer Recurring After Initial Bacillus Calmette-Guérin Therapy. Ann Surg Oncol 2018, 25(8):2484-2491.

5. Hurwitz M, Adeniran A, Yao X, Hafez N, Schalper K, Rimm D, Petrylak D: The effect of BCG intravesical therapy and recurrence on PDL1 expression in non-invasive bladder cancers. Journal of Clinical Oncology 2015, 33:e15504-e15504.

6. Blank C, Gajewski TF, Mackensen A: Interaction of PD-L1 on tumor cells with PD-1 on tumor-specific T cells as a mechanism of immune evasion: implications for tumor immunotherapy. Cancer 
immunology, immunotherapy: Cll 2005, 54(4):307-314.

7. Shi L, Chen S, Yang L, Li Y: The role of PD-1 and PD-L1 in T-cell immune suppression in patients with hematological malignancies. Journal of hematology \& oncology 2013, 6(1):74.

8. Cha JH, Chan LC, Li CW, Hsu JL, Hung MC: Mechanisms Controlling PD-L1 Expression in Cancer. Molecular cell 2019, 76(3):359-370.

9. Sun C, Mezzadra R, Schumacher TN: Regulation and Function of the PD-L1 Checkpoint. Immunity 2018, 48(3):434-452.

10. Tang H, Liang Y, Anders RA, Taube JM, Qiu X, Mulgaonkar A, Liu X, Harrington SM, Guo J, Xin Y et al: PD-L1 on host cells is essential for PD-L1 blockade-mediated tumor regression. The Journal of clinical investigation 2018, 128(2):580-588.

11. Groeger S, Domann E, Gonzales JR, Chakraborty T, Meyle J: B7-H1 and B7-DC receptors of oral squamous carcinoma cells are upregulated by Porphyromonas gingivalis. Immunobiology 2011, 216(12):1302-1310.

12. Groeger S, Jarzina F, Mamat U, Meyle J: Induction of B7-H1 receptor by bacterial cells fractions of Porphyromonas gingivalis on human oral epithelial cells: $\mathrm{B} 7-\mathrm{H} 1$ induction by Porphyromonas gingivalis fractions. Immunobiology 2017, 222(2):137-147.

13. Gollwitzer ES, Saglani S, Trompette A, Yadava K, Sherburn R, McCoy KD, Nicod LP, Lloyd CM, Marsland BJ: Lung microbiota promotes tolerance to allergens in neonates via PD-L1. Nature medicine 2014, 20(6):642-647.

14. Wu P, Zhang G, Zhao J, Chen J, Chen Y, Huang W, Zhong J, Zeng J: Profiling the Urinary Microbiota in Male Patients With Bladder Cancer in China. Frontiers in cellular and infection microbiology 2018, 8:167.

15. Zitvogel L, Daillère R, Roberti MP, Routy B, Kroemer G: Anticancer effects of the microbiome and its products. Nature reviews Microbiology 2017, 15(8):465-478.

16. Dzutsev A, Goldszmid RS, Viaud S, Zitvogel L, Trinchieri G: The role of the microbiota in inflammation, carcinogenesis, and cancer therapy. European journal of immunology 2015, 45(1):1731.

17. Lynch SV, Pedersen O: The Human Intestinal Microbiome in Health and Disease. The New England journal of medicine 2016, 375(24):2369-2379.

18. Willis AD, Minot SS: Strategies to Facilitate Translational Advances from Microbiome Surveys. Trends in microbiology 2020, 28(5):329-330.

19. Ribas A, Wolchok JD: Cancer immunotherapy using checkpoint blockade. Science (New York, NY) 2018, 359(6382):1350-1355.

20. Witjes JA, Bruins HM, Cathomas R, Compérat EM, Cowan NC, Gakis G, Hernández V, Linares Espinós E, Lorch A, Neuzillet $Y$ et al: European Association of Urology Guidelines on Muscle-invasive and Metastatic Bladder Cancer: Summary of the 2020 Guidelines. European urology 2020. 
21. Matson V, Fessler J, Bao R, Chongsuwat T, Zha Y, Alegre ML, Luke JJ, Gajewski TF: The commensal microbiome is associated with anti-PD-1 efficacy in metastatic melanoma patients. Science (New York, NY) 2018, 359(6371):104-108.

22. Routy B, Le Chatelier E, Derosa L, Duong CPM, Alou MT, Daillère R, Fluckiger A, Messaoudene M, Rauber C, Roberti MP et al: Gut microbiome influences efficacy of PD-1-based immunotherapy against epithelial tumors. Science (New York, NY) 2018, 359(6371):91-97.

23. Bi H, Tian Y, Song C, Li J, Liu T, Chen Z, Chen C, Huang Y, Zhang Y: Urinary microbiota - a potential biomarker and therapeutic target for bladder cancer. Journal of medical microbiology 2019, 68(10):1471-1478.

24. Eribe ER, Olsen I: Leptotrichia species in human infections. Anaerobe 2008, 14(3):131-137.

25. Castaño-Rodríguez N, Goh KL, Fock KM, Mitchell HM, Kaakoush NO: Dysbiosis of the microbiome in gastric carcinogenesis. Scientific reports 2017, 7(1):15957.

26. Gao Z, Guo B, Gao R, Zhu Q, Qin H: Microbiota disbiosis is associated with colorectal cancer. Frontiers in microbiology 2015, 6:20.

27. Saffarian A, Mulet C, Regnault B, Amiot A, Tran-Van-Nhieu J, Ravel J, Sobhani I, Sansonetti PJ, Pédron T: Crypt- and Mucosa-Associated Core Microbiotas in Humans and Their Alteration in Colon Cancer Patients. mBio 2019, 10(4).

28. Torres PJ, Fletcher EM, Gibbons SM, Bouvet M, Doran KS, Kelley ST: Characterization of the salivary microbiome in patients with pancreatic cancer. PeerJ 2015, 3:e1373.

29. Warren RL, Freeman DJ, Pleasance S, Watson P, Moore RA, Cochrane K, Allen-Vercoe E, Holt RA: Cooccurrence of anaerobic bacteria in colorectal carcinomas. Microbiome 2013, 1(1):16.

30. Jang JY, Song IS, Baek KJ, Choi Y, Ji S: Immunologic characteristics of human gingival fibroblasts in response to oral bacteria. Journal of periodontal research 2017, 52(3):447-457.

31. Trikha M, Corringham R, Klein B, Rossi JF: Targeted anti-interleukin-6 monoclonal antibody therapy for cancer: a review of the rationale and clinical evidence. Clinical cancer research: an official journal of the American Association for Cancer Research 2003, 9(13):4653-4665.

32. Tanaka T, Narazaki M, Kishimoto T: IL-6 in inflammation, immunity, and disease. Cold Spring Harbor perspectives in biology 2014, 6(10):a016295.

33. Chan LC, Li CW, Xia W, Hsu JM, Lee HH, Cha JH, Wang HL, Yang WH, Yen EY, Chang WC et al: IL6/JAK1 pathway drives PD-L1 Y112 phosphorylation to promote cancer immune evasion. The Journal of clinical investigation 2019, 129(8):3324-3338.

34. Calcinotto A, Brevi A, Chesi M, Ferrarese R, Garcia Perez L, Grioni M, Kumar S, Garbitt VM, Sharik ME, Henderson KJ et al: Microbiota-driven interleukin-17-producing cells and eosinophils synergize to accelerate multiple myeloma progression. Nature communications 2018, 9(1):4832.

35. Zhang M, Fan X, Fang B, Zhu C, Zhu J, Ren F: Effects of Lactobacillus salivarius Ren on cancer prevention and intestinal microbiota in 1, 2-dimethylhydrazine-induced rat model. Journal of microbiology (Seoul, Korea) 2015, 53(6):398-405. 
36. Myles IA, Earland NJ, Anderson ED, Moore IN, Kieh MD, Williams KW, Saleem A, Fontecilla NM, Welch PA, Darnell DA et al: First-in-human topical microbiome transplantation with Roseomonas mucosa for atopic dermatitis. JCl insight 2018, 3(9).

37. Colliou N, Ge Y, Sahay B, Gong M, Zadeh M, Owen JL, Neu J, Farmerie WG, Alonzo F, 3rd, Liu K et al: Commensal Propionibacterium strain UF1 mitigates intestinal inflammation via Th17 cell regulation. The Journal of clinical investigation 2017, 127(11):3970-3986.

38. Trowitzsch S, Tampé R: ABC Transporters in Dynamic Macromolecular Assemblies. Journal of molecular biology 2018, 430(22):4481-4495.

39. Lövgren T, Eloranta ML, Båve U, Alm GV, Rönnblom L: Induction of interferon-alpha production in plasmacytoid dendritic cells by immune complexes containing nucleic acid released by necrotic or late apoptotic cells and lupus IgG. Arthritis and rheumatism 2004, 50(6):1861-1872.

40. Jacquelot N, Yamazaki T, Roberti MP, Duong CPM, Andrews MC, Verlingue L, Ferrere G, Becharef S, Vétizou M, Daillère R et al: Sustained Type I interferon signaling as a mechanism of resistance to PD1 blockade. Cell research 2019, 29(10):846-861.

41. Caporaso JG, Kuczynski J, Stombaugh J, Bittinger K, Bushman FD, Costello EK, Fierer N, Peña AG, Goodrich JK, Gordon JI et al: QIIME allows analysis of high-throughput community sequencing data. Nature methods 2010, 7(5):335-336.

42. Zhang J, Liu YX, Zhang N, Hu B, Jin T, Xu H, Qin Y, Yan P, Zhang X, Guo X et al: NRT1.1B is associated with root microbiota composition and nitrogen use in field-grown rice. Nature biotechnology 2019, 37(6):676-684.

43. Magoč T, Salzberg SL: FLASH: fast length adjustment of short reads to improve genome assemblies. Bioinformatics (Oxford, England) 2011, 27(21):2957-2963.

44. Edgar RC: UPARSE: highly accurate OTU sequences from microbial amplicon reads. Nature methods 2013, 10(10):996-998.

45. Rognes T, Flouri T, Nichols B, Quince C, Mahé F: VSEARCH: a versatile open source tool for metagenomics. PeerJ 2016, 4:e2584.

46. Edgar RC: Search and clustering orders of magnitude faster than BLAST. Bioinformatics (Oxford, England) 2010, 26(19):2460-2461.

47. Quast C, Pruesse E, Yilmaz P, Gerken J, Schweer T, Yarza P, Peplies J, Glöckner FO: The SILVA ribosomal RNA gene database project: improved data processing and web-based tools. Nucleic acids research 2013, 41(Database issue):D590-596.

48. DeSantis TZ, Hugenholtz P, Larsen N, Rojas M, Brodie EL, Keller K, Huber T, Dalevi D, Hu P, Andersen GL: Greengenes, a chimera-checked 16S rRNA gene database and workbench compatible with ARB. Applied and environmental microbiology 2006, 72(7):5069-5072.

49. Wang Q, Garrity GM, Tiedje JM, Cole JR: Naive Bayesian classifier for rapid assignment of rRNA sequences into the new bacterial taxonomy. Applied and environmental microbiology 2007, 73(16):5261-5267. 
50. Letunic I, Bork P: Interactive Tree Of Life v2: online annotation and display of phylogenetic trees made easy. Nucleic acids research 2011, 39(Web Server issue):W475-478.

51. Segata N, Izard J, Waldron L, Gevers D, Miropolsky L, Garrett WS, Huttenhower C: Metagenomic biomarker discovery and explanation. Genome biology 2011, 12(6):R60.

52. Chong J, Liu P, Zhou G, Xia J: Using MicrobiomeAnalyst for comprehensive statistical, functional, and meta-analysis of microbiome data. Nature protocols 2020, 15(3):799-821.

53. Langille MG, Zaneveld J, Caporaso JG, McDonald D, Knights D, Reyes JA, Clemente JC, Burkepile DE, Vega Thurber RL, Knight $\mathrm{R}$ et al: Predictive functional profiling of microbial communities using $16 \mathrm{~S}$ rRNA marker gene sequences. Nature biotechnology 2013, 31(9):814-821.

54. Parks DH, Tyson GW, Hugenholtz P, Beiko RG: STAMP: statistical analysis of taxonomic and functional profiles. Bioinformatics (Oxford, England) 2014, 30(21):3123-3124.

\section{Supplementary}

Supplementary Table S1-S2 and Figures S1-S3 are not available with this version

\section{Figures}
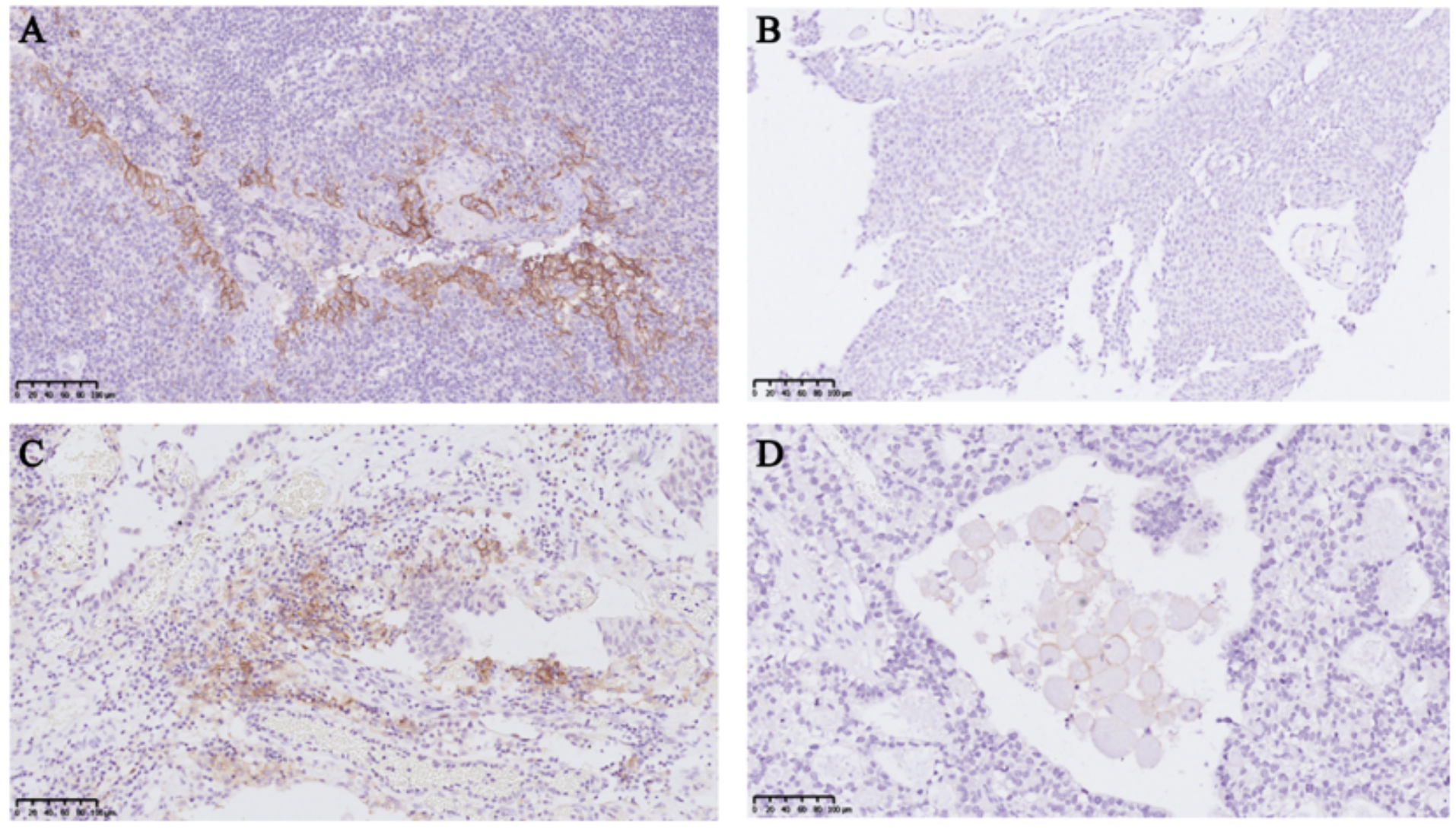

Figure 1 
PD-L1 expression in formalin-fixed paraffin-embedded tissue samples stained with anti-PD-L1 antibody (405.9A11). Positive control with tonsil tissue for PD-L1 expression is presented in panel (A). In panel (B), both tumor cells and TAMs are negative for PD-L1. Positive membranous staining in tumor cells and TAMs are presented in panels $(C)$ and $(D)$, respectively
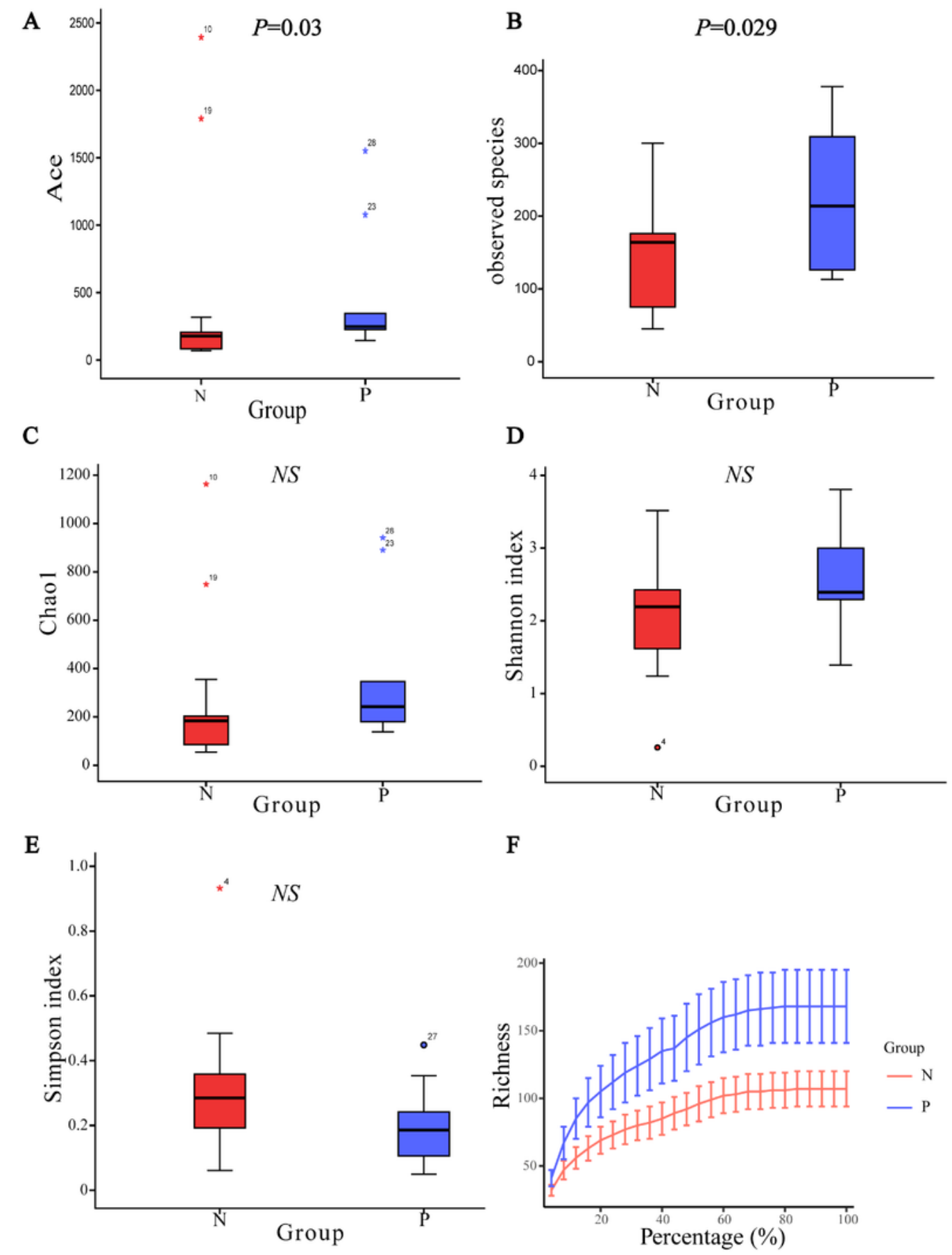

\section{Figure 2}


Alpha Diversity and Alpha rarefaction curves for PD-L1-positive (P group) and PD-L1-negative groups (N group). Ace index (A), observed species (B), Chao1 index (C), Shannon index (D) and Simpson index (E) were used for the assessment of alpha diversity between $\mathrm{P}$ group and $\mathrm{N}$ group. Rarefaction curves show that the Richness of $\mathrm{P}$ group was much higher than that of $\mathrm{N}$ group $(\mathrm{F})$.

A

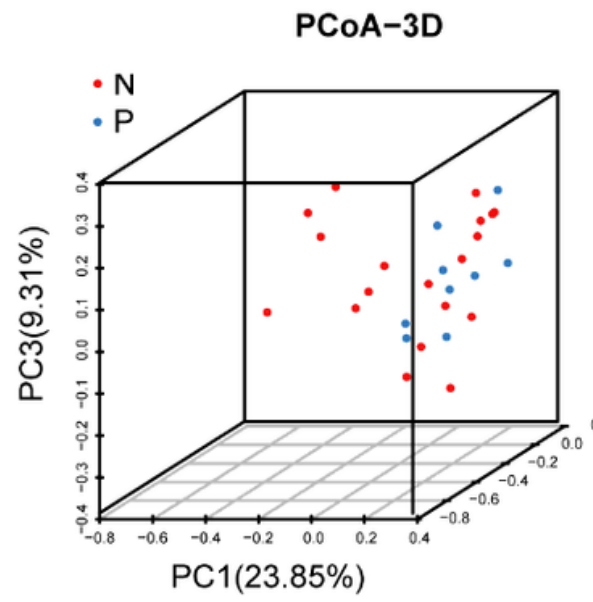

B

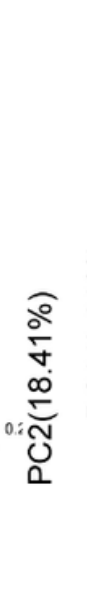

B

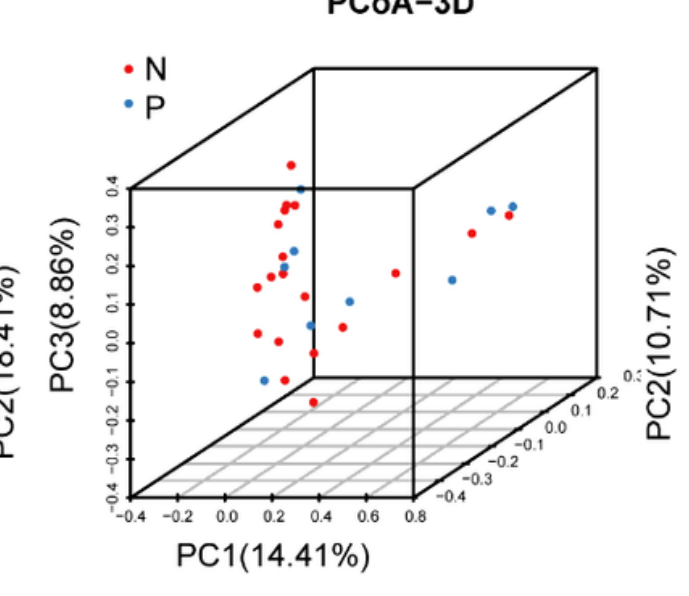

C

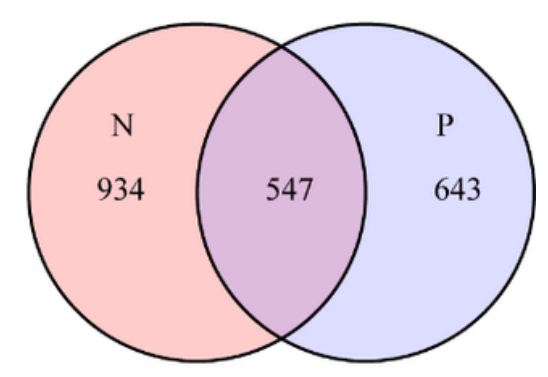

Figure 3

The composition of urinary microbiota in $\mathrm{P}$ group differed from that in $\mathrm{N}$ group. Principal coordinate analysis ( $P C O A)$ of weighted UniFrac distance $(A)$ and unweighted UniFrac distance $(B)$ show that the urinary microbiota of $P$ group and $N$ group formed two distinct clusters $(P<0.05)$. Venn diagrams $(C)$ depict different OTU composition between $\mathrm{N}$ group and $\mathrm{P}$ group. 
A

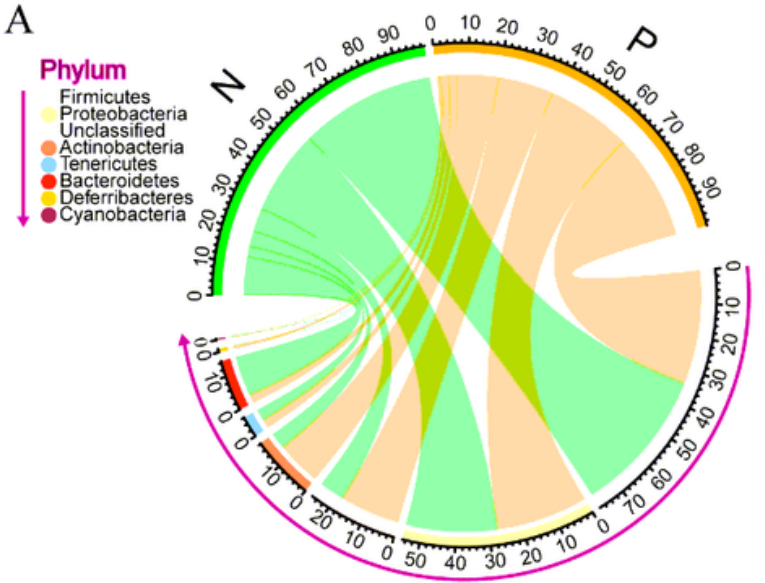

C Order

- Lactobacillales

C Corynebacteriales

Pseudom
Bacillales

Selenomonadale

Enterobacteria
Pasteurellales

:

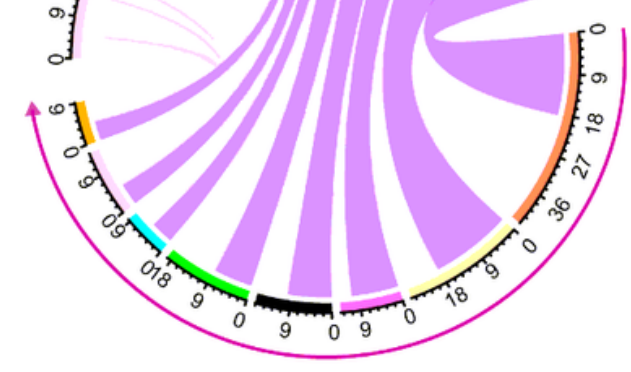

E

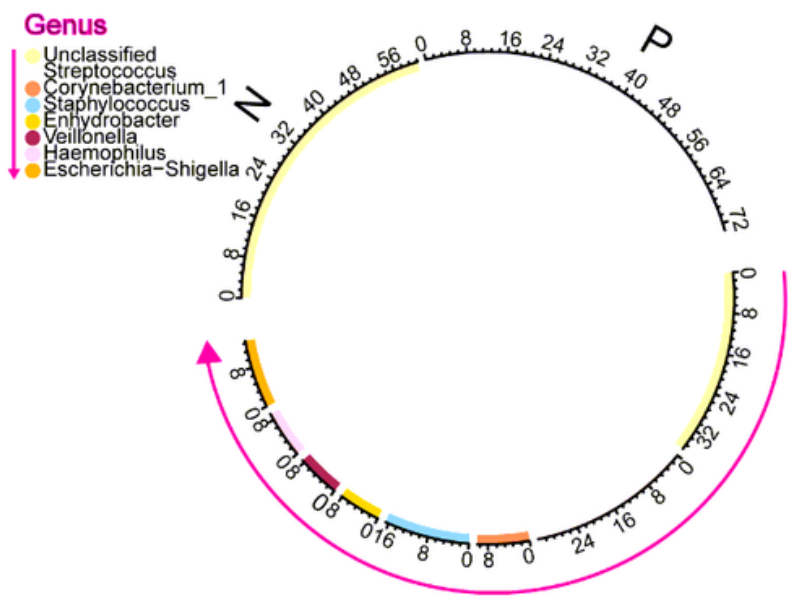

B
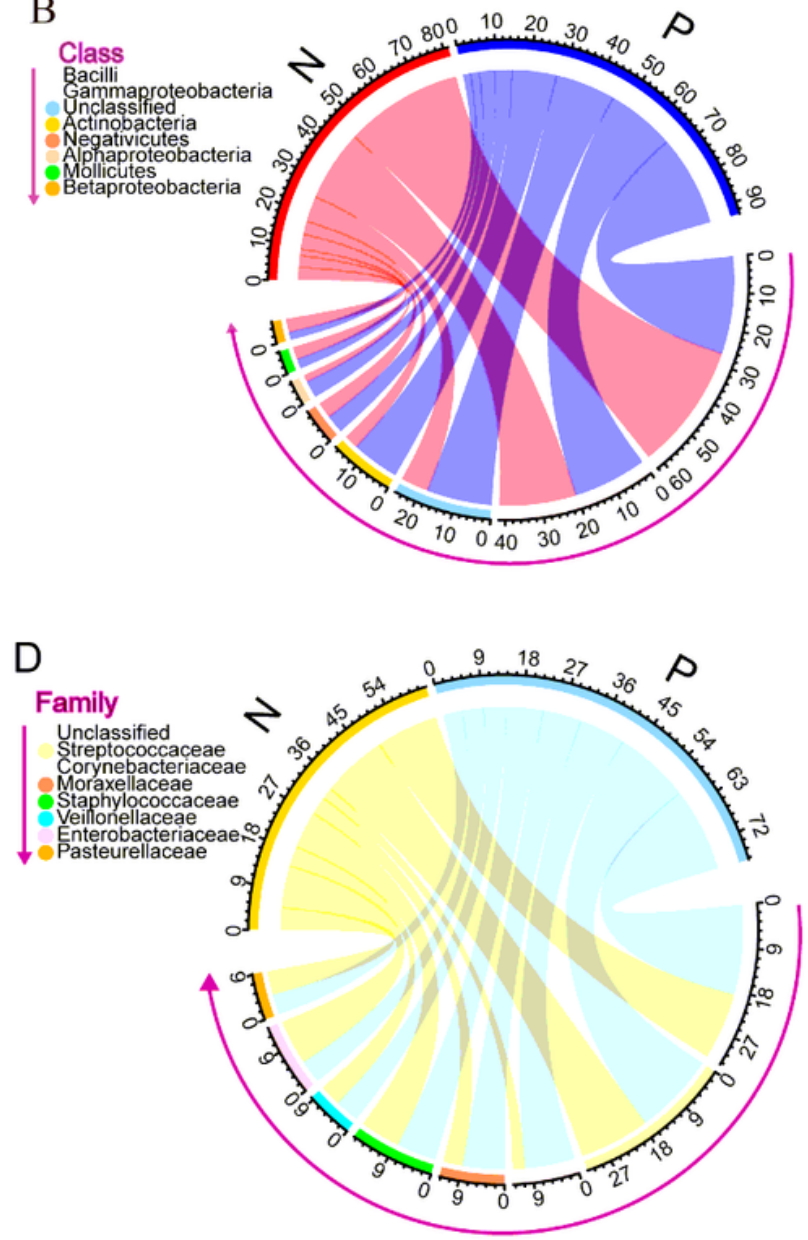

F

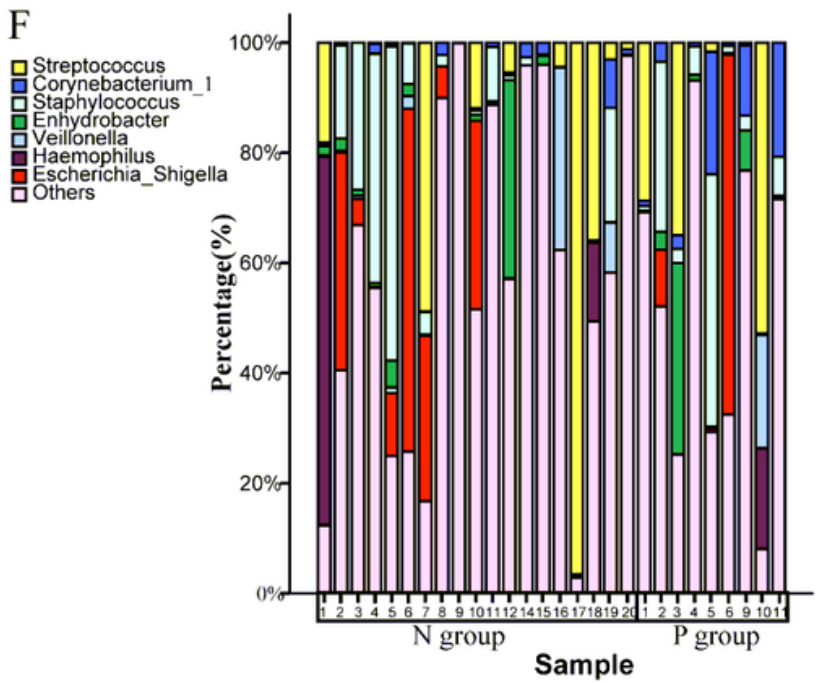

Figure 4

Bacterial relative abundance in PD-L1 positive group and PD-L1 negative group. Average distributions of 8 major taxa are represented by circlize graphs at the level of Phylum (A), Class (B), Order(C), Family (D) and Genus (E). Each color represents a bacterial taxon which is displayed sequentially in the direction indicated by the arrow and the width of a colored ribbon represents the relative abundance of that organism within the sample. In percentage bar chart (F), each bar represents a subject and each colored 
box, a bacterial taxon. The height of a colored box represents the relative abundance of that organism within the sample.

Tree scale: 1

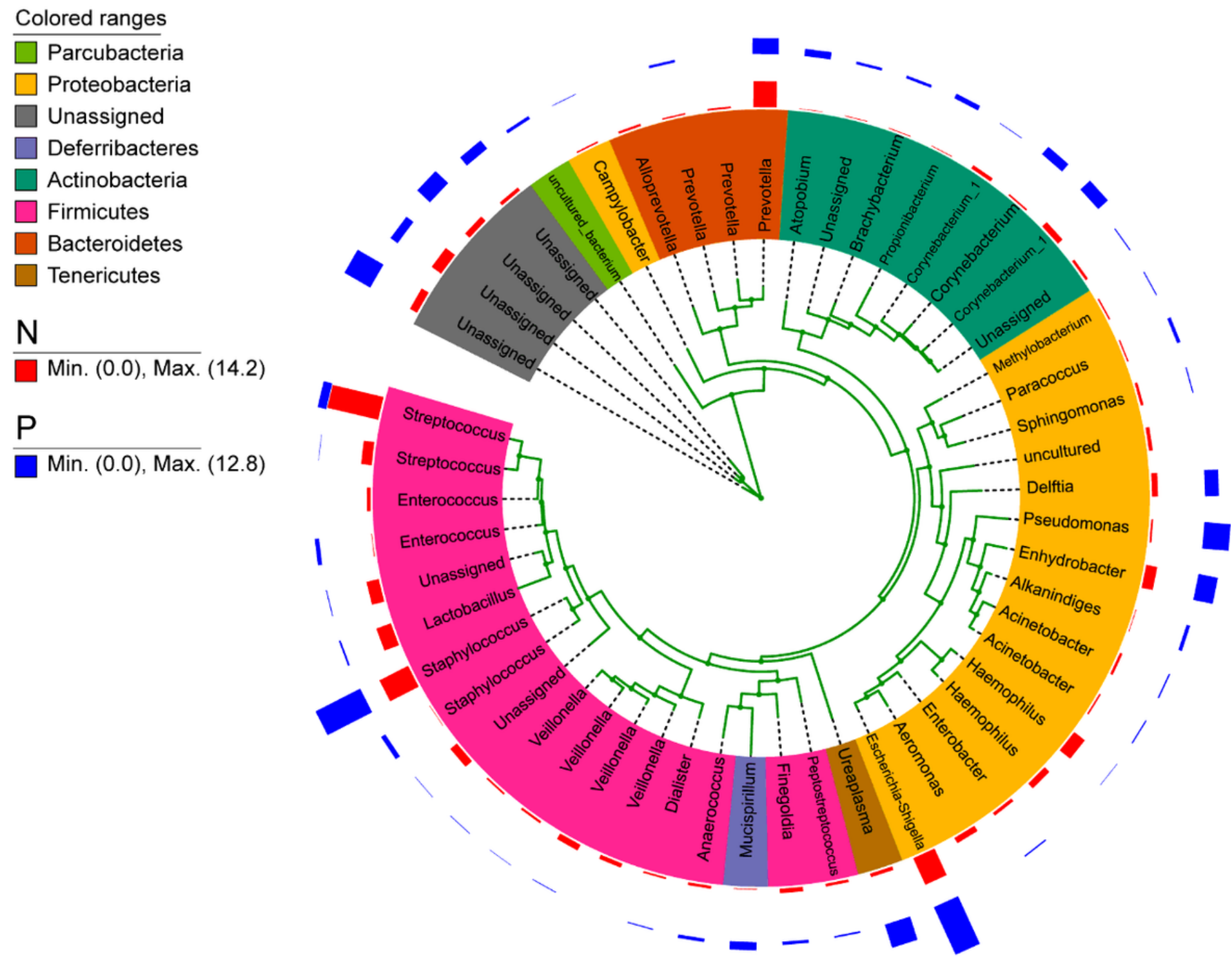

Figure 5

The core microbes associated with the $\mathrm{N}$ group and $\mathrm{P}$ group. The core microbes of each group are displayed by taxonomic dendrogram. Color ranges identify phyla within the tree. Colored bars represent the relative abundance of each OTU in different groups. 
A

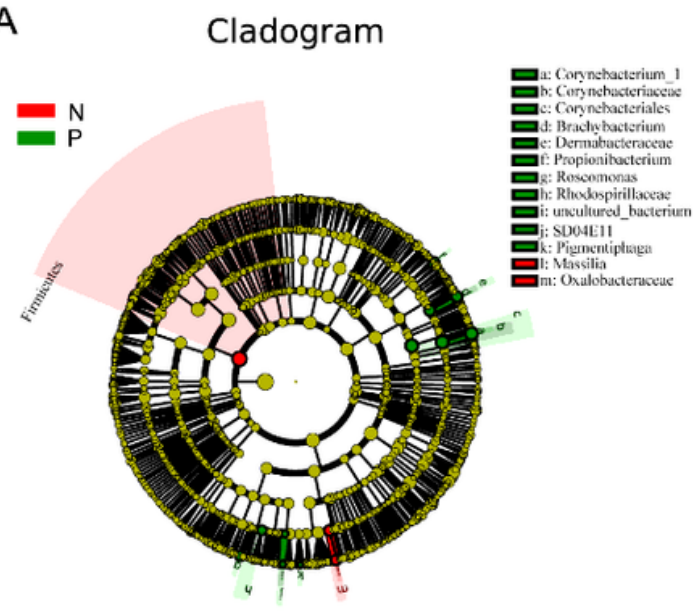

B

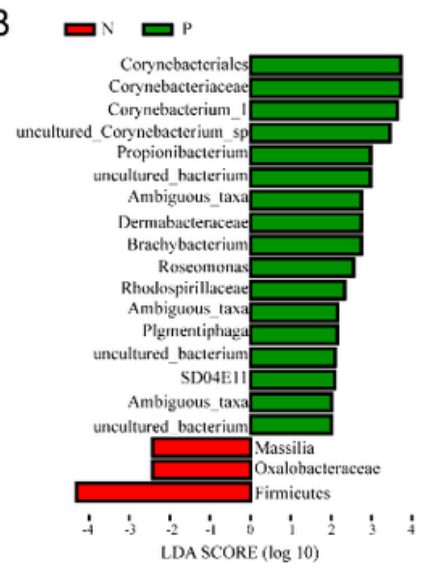

C

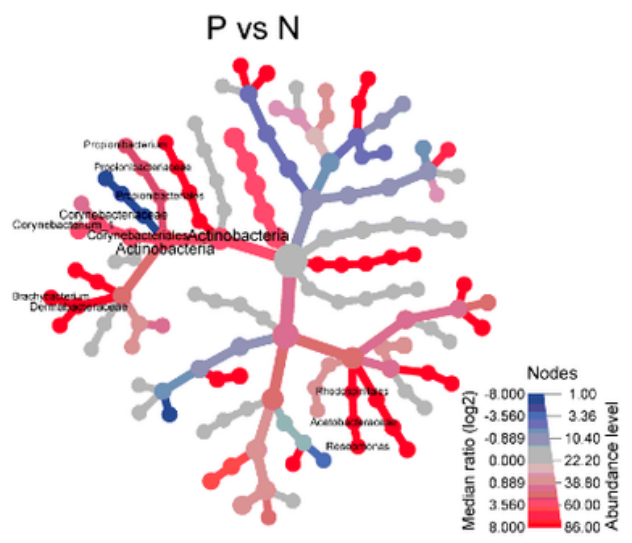

Figure 6

Specific taxa associated with PD-L1 expression. The urinary microbial taxa associated with PD-L1 positivity (green) and PD-L1 negativity (red) are revealed in Cladogram (A). Association of specific microbial taxa with PD-L1 positive and PD-L1 negative group is exhibited by linear discriminant analysis effect size (LEfSe) (B).Green indicates taxa enriched in PD-L1 positive group and red indicates taxa enriched in PD-L1 negative group. Heat tree is used to illustrate the taxonomic differences between $\mathrm{N}$ group and $\mathrm{P}$ group $(\mathrm{C})$. The color gradient and the size of node, edge and label are based on the log2 ratio of median abundance. Blue and red indicate that corresponding taxa are lower and higher, respectively, in $\mathrm{P}$ group as compared with $\mathrm{N}$ group. 